(C) 2015 IEEE. Personal use of this material is permitted. Permission from IEEE must be obtained for all other uses, in any current or future media, including reprinting/republishing this material for advertising or promotional purposes, creating new collective works, for resale or redistribution to servers or lists, or reuse of any copyrighted component of this work in other works. 


\title{
Zeroforcing Precoding based MAC design to address Hidden Terminals in MU-MIMO WLANs
}

\author{
Sanjeeb Shrestha*, Gengfa Fang*, Eryk Dutkiewicz* and Xiaojing Huang ${ }^{\dagger}$ \\ *Department of Engineering, Macquarie University \\ Sydney, Australia \\ ${ }_{\dagger}$ UTS, Australia \\ Email: sanjeeb.shrestha@mq.edu.au
}

\begin{abstract}
This paper focuses on the Medium Access Control (MAC) layer design for an inevitable Hidden Terminal problem in Multi User Multiple Input Multiple Output (MU-MIMO) Wireless Local Area Networks (WLANs). Specifically, our MAC design is supported by the precoding vectors obtained by Zeroforcing technique which are used to address the Hidden Terminals. An efficient channel sounding process is used by our MAC protocol to obtain the Channel State Information (CSI) from the desired and undesired clients which are used to calculate the precoding vectors at the transmitters (Access Points). Our MAC design then uses these precoding vectors in order to null interferences among the undesired clients to avoid collision of signals and to maintain the concurrent transmissions among the desired clients. The the parameters such as network capacity, signaling overheads and fairness are considered in the design.

Our MAC layer design shows a slightly higher signaling overhead compared to RTS/CTS scheme. However, due to the concurrent transmissions after the handshaking process, the cost of singling overheads are compensated. The simulation study of our MAC layer design shows a remarkable constant network capacity gain of 4-5 times in comparison to traditional RTS/CTS. Moreover, the gain is irrespective to the available air-time.
\end{abstract}

Keywords-Hidden Terminals, Precoding Vector, Nulling, Transmission opportunity, Fairness.

\section{INTRODUCTION}

Unlicensed spectrum backed with inexpensive access points and easy deployment have made wireless networks under the IEEE802.11standard almost ubiquitous (e.g. in home, workplace, college campuses, parks etc). This trend is to continue in years to come [1] due to the enterprise dependency on Wireless Local Area Networks (WLANs) for mission critical networks, the growing use of multimedia services with heterogeneous hardware such as iphones, ipads, tablets etc., and the Bring Your Own Device (BYOD) trend. As a result, WLANs have become dynamic in topologies, complex in irregular traffic pattern and challenging from the architectural view point. In this context, collisions of frames are inevitable. Cheng et. al showed that transmission loss due to interference among $50 \%$ of sender receiver pairs suffers $2.5 \%$ probability of transmission loss [2]. While IEEE802.11 CSMA/CA with RTC/CTS has become a de-facto mechanism to avoid collisions, there exist inherent limitations as to how it treats interference at the receiver related to the carrier sensing at the transmitter. However, the fact is that successful transmissions mostly depend on the interference free condition at the receiver.
Theoretical and experimental works on CSMA/CA [3][4] showed that CSMA/CA mechanism degrades performance due to poor spatial reuse and also fails to address the Hidden Terminal (HT) [5] and the capture effect [6] issues. However, HT nodes (that do not sense each others transmission though they interfere with each other at the intended receiver causing decoding failure) is an inevitable phenomena in WLANs. Additionally, the impact of HTs is significant. The study in [7] reveals that HTs lead to about $40-42 \%$ of collision loss.

In early years, a receiver initiated busy tone scheme was proposed to solve the HT problem for Packet Radio Networks (PRN) which was found to be effective in eliminating collisions caused by HTs [5]. However, the scheme required a dedicated channel for the busy tone which is not desirable in wireless networks. Later, Karn proposed the RTS/CTS mechanism as a part of MACA [8] to address the HT problem, however, experimental results show that RTS/CTS significantly reduces the overall throughput [9] and is disabled by Access Point (AP) manufactures by default. A recent study proposed a lightweight wireless handshake [10] where the header of the payload and ACK are separated and designed to act like RTS/CTS. However, packet decoding in dynamic channels is a fundamental question for that approach. Addressing the HT in WLANs using CDMA is not viable as it requires tight power control and special codes [11] and at high SNRs the performance is degraded. An alternative technique like zigzag decoding [12] analyzes collisions of packets with strategically selected collision patterns, showing a significant packet reduction loss from $72.06 \%$ to about $0.7 \%$. However, it needs to have a collision free chunk to bootstrap decoding in an irregular traffic pattern such as in WLAN scenarios. Besides, the scheme works only for certain type of collision patterns, thus it is practically limited.

We present the Medium Access Control (MAC) layer design to deal with the HT problem. Unlike its precursors [5][8][10][12], our MAC design uses the precoding vectors calculated by Zeroforcing in order to get rid of collisions loss in the HT scenario. Meanwhile, our MAC layer design takes care of fairness and maintains a constant capacity gain with respect to RTS/CTS despite signaling overhead.

For instance, lets take an example of the $i$ th network, AP2, and the $j$ th network, AP1, in Fig.1. Due to the nature of dynamic topologies, non-isotropic nature of the wireless 


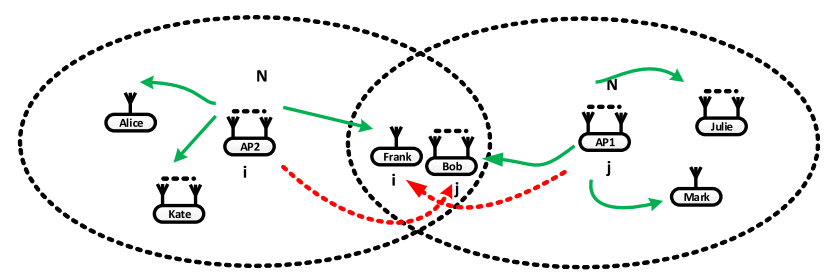

Fig. 1. Hidden Terminal

transmission range, mix-mode $802.11 \mathrm{~b} / \mathrm{g} / \mathrm{n}$ usages, dense deployments, decentralized control etc., they are both out of the carrier sensing range of each other but their transmissions overlap. Thus, AP1 and AP2 are Hidden Terminals to each other. When the APs transmit to their desired clients (AP2 to 'Frank' and AP1 to 'Bob'), the undesired clients ('Bob' for AP2 and 'Frank' for AP1) suffer collision of signals from their respective Hidden APs i.e., AP2 for 'Bob' and AP1 for 'Frank'. The green and the dotted red arrow indicate the desired and the undesired signals respectively.

From high level view, our proposed scheme makes AP2 null its signal at 'Bob' while transmitting to 'Frank' so that AP1 can transmit to 'Bob'. Similarly, AP1 nulls its signal to 'Frank' while transmitting to 'Bob' so that AP2 can transmit to 'Frank' simultaneously. Specifically, we use the precoding vectors at both the APs to null the signal at the undesired clients while it is transmitting to the desired client. In this scenario neither of the APs have to listen and wait before transmission as in the case when using RTS/CTS nor the receiver clients do have to re-encode any former decoded chunks as in the Successive Interference Cancellation (SIC) scheme.

The rest of the paper is arranged as follows: Section II presents the System model whereas Section III illustrates the Physical Layer (PHY). Section IV describes the MAC layer and Section $\mathrm{V}$ presents the performance evaluation of our protocol. In Section VI conclusion is presented.

Notation:The superscript $(.)^{H}$ denotes the Hermitian transpose whereas the operators $\mathrm{E}[$.$] and \|$.$\| denote expectation$ and the Euclidean norm respectively. The matrices, vectors and scalars are defined next, as they are used.

\section{SYSTEM MODEL}

We present our system model for $K$ Access Points (APs). The number of antenna at APs and the client are considered to be $N$ and $M$ respectively. Now, the received signal at a client in the overlapping (HT) region is given by

$$
\mathbf{y}=\sum_{i=1}^{K} \mathbf{h}_{i j}^{H} \mathbf{x}_{i}+\mathbf{w}
$$

where the received signal is $\mathbf{y} \in \mathbb{C}^{M \times 1}, \mathbf{h}_{i j}$ is the channel associated with the $i$ th AP to the $j$ th Client, $\mathbf{h}_{i j} \in \mathbb{C}^{N \times M}$ and transmitted signal $\mathbf{x}_{i} \in \mathbb{C}^{N \times 1}$. The noise term is represented by $\mathbf{w} \in \mathbb{C}^{M \times 1}$ which is circularly symmetric additive white Gaussian noise with zero mean and $\sigma^{2}$ variance. All the APs satisfy a transmit power constraint $P$, i.e., $E\left\|\mathbf{x}_{i}^{2}\right\|<$ $P$, for $i=1 \ldots . . K$. The concatenation of channels at the $j$ th client is given by

$$
\mathbf{H}=\left[\mathbf{h}_{1 j}^{H}, \mathbf{h}_{2 j}^{H}, \ldots . \mathbf{h}_{j j}^{H}, \ldots . . \mathbf{h}_{K-1 j}^{H}, \mathbf{h}_{K j}^{H}\right]
$$

where $\mathbf{H}$ is a $[M \times K N]$ matrix with the $j$ th row equal to the channel of the $K$ APs to the $j$ th antenna elements of the $j$ th client with $M$ antennas.

\section{The PhysicAl LAYER}

The physical layer of our protocol is developed to achieve high network throughput by multiple simultaneous transmissions without interferences to others (Here-forth, we mention concurrent transmissions for the same) in a network. Traditional protocols in WLANs work on time sharing basis and is unable to do so. We develop our physical layer from both time and space shared approach provided by the multiple antennas at the APs and clients.

Specifically, we used signal nulling technique carried out by $K-1$ APs to remove collision of signal at a client. For simplicity, we discuss collision of signals with reference to Fig. 1 where two APs, AP1 and AP2 (i.e $\mathrm{K}=2$ ), and the $j$ th reference client 'Bob' are taken into consideration.

The physical layer solution, nulling, is used by AP2 to null its signal at 'Bob' when it is transmitting to other clients. The nulling of signal by AP2 is assisted by the precoding vector. As a matter of fact, the calculation of precoding vector at AP2 is vital as it not only removes interferences to undesired client but also maintains concurrent transmissions among the desired clients.

\section{A. Precoding Vector}

We use Zeroforcing in order to calculate precoding vectors at the APs in our network. The precoding vectors at APs are such that they maximize the desired transmissions, meanwhile null interferences at the undesired clients. The choice for the best $\mathrm{ZF}$ beamforing vector for any $i$ th AP2 is given by sloving the following optimization problem for $i \in\{1, \ldots \ldots . K\}$

$$
\begin{gathered}
\max _{\mathbf{v}_{i}} \log \left(1+\frac{\left\|\mathbf{h}_{i i}^{H} \mathbf{v}_{i}\right\|^{2}}{\sigma_{i}^{2}}\right) \\
\text { s.t } \quad\left\|\mathbf{h}_{i j}^{H} \mathbf{v}_{i}\right\|^{2}=0 \forall j \neq i \\
\left\|\mathbf{v}_{i}\right\|^{2} \leqslant P_{i}
\end{gathered}
$$

where $\left\|\mathbf{h}_{i j}^{H} \mathbf{v}_{i}\right\|^{2}=0$ is the ZF leakage constraint of the $i$ th AP2 to clients $j$. The optimization problem has the non-trivial solution given by $\mathbf{v}_{i}^{Z F}=c \prod_{\left[h_{i 1}, \ldots \ldots . h_{i i-1}, h_{i i+1} \ldots \ldots h_{i K}\right]}^{\perp} \mathbf{h}_{i i}$, where $c$ is the scalar satisfying the transmit power constraint. The necessary condition for the non-trivial solution is $N>M$. Specifically, the precoding vector for AP2, $\mathbf{v}_{i} \in \mathbb{C}^{N \times 1}$, is given by

$$
\mathbf{v}_{i}^{z f}=\left(\frac{\prod_{\mathbf{h}_{i j}}^{\perp} \mathbf{h}_{i i}}{\left\|\prod_{\mathbf{h}_{i j}}^{\perp} \mathbf{h}_{i i}\right\|} \mathbf{U}\right)
$$

where $\prod_{\mathbf{h}_{i j}}^{\perp}=\mathbf{I}-\mathbf{h}_{i j}\left(\mathbf{h}_{i j}{ }^{H} \mathbf{h}_{i j}\right)^{-1} \mathbf{h}_{i j}{ }^{H}$ denotes projection onto the orthogonal complement of the column space of $\mathbf{h}_{i j}$. 
$\mathbf{I}_{N}$ represents the identity matrix of size $N$ (the subscript is omitted when unnecessary). $\mathbf{U} \in \mathbb{C}^{M \times 1}$ is a unit vector acting as a demultiplexer where $\mathbf{U}^{H} \mathbf{U}=1$.

It is worthwhile to note that the expression in Eq. 6 gives a theoretical description of the precoding vector in reference to the $i$ th network AP2 and the $j$ th network client, 'Bob'. However, in a general network scenario, where there can be mix match among the desired and undesired clients, there is a need to make a concurrent transmissions without interfering with each other. In such context, the expression for precoding vector in Eq.6 still remains valid, except that we deal with $N \times P M$ channel realizations for $P$ undesired (i.e $\mathbf{h}_{i j}$ ) clients and $N \times Q M$ channel realizations for $Q$ desired clients (i.e $\mathbf{h}_{i i}$ ). The condition $N>P M$ has to be satisfied in order to take the left inverse. ${ }^{1}$

In fact, in a network scenario of heterogeneous clients, first of all, the CSI associated with the desired and undesired clients are obtained via channel sounding. The detail process of channel sounding is provided in Section $I V, A$. Second, the channels associated with the undesired clients and the desired clients are identified by the Association Identification (AID) assign to them by the APs. The detail description about the identification is on section SectionIV. The channels to undesired clients i.e $N \times P M$ realizations are used to form a complementary projection matrix $\prod_{\mathbf{h}_{i j}}^{\perp}$. This projection matrix is then used by the APs to project the channels associated with the desired clients (i.e $N \times Q M$ ) into the complementary orthogonal space of $N \times P M$ channel realizations for $P$ undesired clients. Ultimately, this resembles the solution of the optimization problem present in 3 .

From the standpoint of the $j$ th client 'Bob', the received signal is given by

$$
\underbrace{\mathbf{y}_{j}}_{\text {received signal }}=\underbrace{\mathbf{h}_{j j}^{H} \mathbf{v}_{j} s_{j}}_{\text {desired signal }}+\underbrace{\sum_{i=1, i \neq j}^{K-1} \mathbf{h}_{i j}^{H} \mathbf{v}_{i} s_{i}}_{\text {interferences }}+\underbrace{\mathbf{w}_{j}}_{\text {noise }} .
$$

The pictorial presentation of the signal spaces is given in

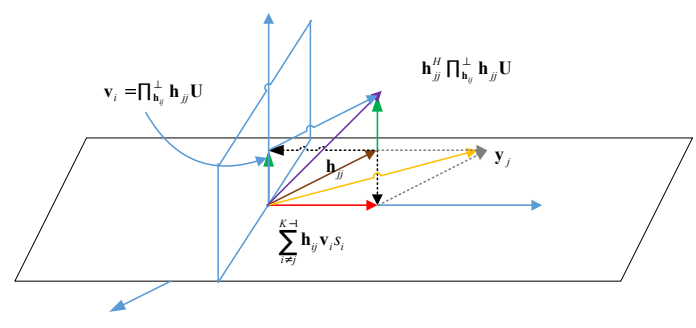

Fig. 2. Signal Space of the received signal of 'Bob'

Fig. 2 where the yellow, brown, red and purple arrows indicate the received, desire, interference and the maximized signals respectively. Since $\mathbf{v}_{i}$ is calculated with Eq.6, it implies that $\mathbf{h}_{i j}^{H} \mathbf{v}_{i} s_{i}=0$ and $\mathbf{h}_{j j}^{H} \mathbf{v}_{j} s_{j}$ is maximized. Hence, the desired signal is received by 'Bob'.

\footnotetext{
${ }^{1}$ The right inverse can still be taken if $N<P M$, however this will not increase the concurrent transmissions as the number of degree of freedom of the network is $\min \{\mathrm{N}, \mathrm{PM}\}$
}

\section{Medium AcCess Control}

Since the physical layer solution of our protocol allows multiple APs to transmit concurrently to their desired clients without interferences to others, we need to develop the MAC protocol suited for this. The challenge is three folds. First, acquiring the CSI associated with the transmitter and clients and obtain the procoding vector (steering vector) for concurrent transmissions. Second, the signalling overhead (i.e handshaking process) for completing the first process should be as minimal as possible for capacity increment of the network. Third, the fairness in transmission should be maintained among the APs with heterogeneous antennas. Other important aspects are to move the complexity of signal processing to the APs and dynamically change the combination of concurrent clients in bursty traffic demand, while respecting fairness. We shall discuss these aspects further.

\section{A. Acquiring CSI associated with transmitter and clients}

The channel sounding is initiated by those APs, who have packets in queue for transmission. Since the APs need to find the channels associated with desired and undesired clients, they first broadcast an announcement frame so that the clients within the APs' transmission range can report their channels to the APs. The frames formats used in channel sounding is shown in Fig.3. The NDP announcement control frames of 25 bytes in length are transmitted by the APs. The frame contains 6 bytes field for transmitter address (i.e AP) and separate address fields for a set of multiple stations information records used to request multi-user feedback. Most importantly, APs assign an Association Identifications (AIDs) to the clients upon association which are included in the 12 Least Significant Bits (LSB).

The second step is to send the training symbols by the APs for channel measurement. This is done by the Null Data Packet (NDP) frames which have the same format as VHT PPDUs without data field. Each client analyzes the training symbols in the PLCP header (of the NDP) and measures the channel between the APs and themselves. It is obvious that the clients within the overlapping region would hear multiple NDP announcements. Each client respond to the channel request with First In First Out (FIFO) basis in the uplink. After the reception of the NDPs, the third step is to feedback the measured channels. Owing to the limited feedback channel, the channels (in the form of the matrices) are compressed and sent in the form of a VHT Compressed Beamforming frames. Since the APs need to differentiate the channels associated with the desired and undesired clients, the clients having AIDs with the APs will only respond with VHT Compressed Beamforming Frames and the remaining clients wait further to be polled by the APs.

Fourth, the APs sent the Beamforming Report Poll frames after SIFS with the view to know whether or not there are any other clients in their range. This 12 bytes long control frames poll and retrieve the additional channel matrices in the form of the VHT Compressed Beamforming frames from the subsequent clients within the range. Each bit in the 
feedback bitmap field requests one feedback segment to be retransmitted. Hence, by the end of the channel sounding all the APs in the network will get the channels from the desired and the undesired clients within the range. In terms of power, regulation can limit the transmit power based on the number of antennas used at the transmitter so that transmit beamforming does not increase the maximum distance range.

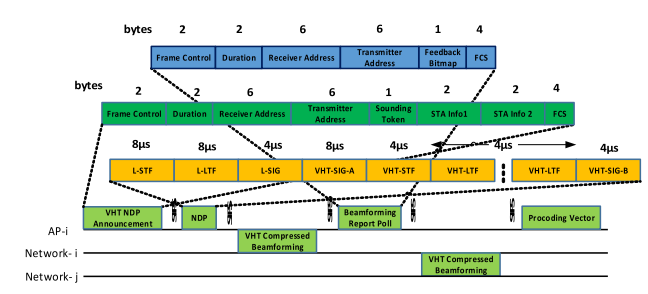

Fig. 3. Frames formats suited for our protocol for channel measurement

Fig. 3 represents a basic diagram of the frames formats and the channel measurement process for the APs in a typical $i$ th network, AP2 and two clients, each from the $i$ th and $j$ th networks.

Upon the reception of all the channels the APs, the precoding vector for concurrent transmissions are calculated according to the PHY solution presented in Eq.6.

As a matter of fact, in the typical scenario considered, the precoding vector is the master steering vector required for concurrent transmissions by the AP2 among its clients 'Kate', 'Alice' and 'Frank', without interfering 'Bob'.

\section{B. Transmission opportunity for APs with heterogeneous an- tennas}

All the APs in the network have to calculate their corresponding precoding vectors for concurrent transmissions. Obtaining such precoding vectors are possible only when the APs who are willing to transmit, do have available degrees of freedom to do so. ${ }^{2}$ Otherwise, the AP ends up with causing interference with other clients. Suppose that we have 3 APs in a network having $N_{1}, N_{2}$ and $N_{3}$ antennas respectively. Each of these AP has $I_{1}, I_{2}, I_{3}$ clients in the overlapping region. The three APs satisfying $N_{1}>I_{2}+I_{3}, N_{2}>I_{1}+I_{3}$ and $N_{3}>I_{1}+I_{2}$ will have transmission opportunity (TXOP) and will remain in the 'Active' mode, otherwise, the APs will not have TXOP and remain in the 'Silent' mode at that instant. Thus, the TXOP among the APs are decided on the available degree of freedom at particular AP at the particular time. This process is coordinated by a beacon which sets a fix transmission inverval. The beacons are transmitted by all the APs who want the TXOP.

After the transmission of beacon, channel sounding is initiated, upon its completion, TXOP is decided. After getting the TXOP, the APs calculate the precoding vectors and start concurrent transmissions untill the duration of beacon. The

\footnotetext{
${ }^{2}$ For instance, in a network of 3 ongoing concurrent transmissions, a two antenna AP does not qualify for transmission opportunity (TXOP), however, the APs with greater number of antennas than 3 can obtain the precoding vector and can transmit concurrently.
}

APs, who fail to win the TXOP, remains in the 'Silent' mode and continuously monitor the beacon duration and waits for next TXOP.

It appears that this criteria for the TXOP, favors APs with larger number of antennas resulting in a deep unfairness among the APs having less numbers of antennas.

In order to ensure the fairness among the APs with heterogeneous antenna, we assign all the APs with two type of credit counters ('SCounter' and 'FCounter') and a credit threshold ('Cthreshold'). Each time when APs get the TXOP, the 'Scounter' counter will be incremented otherwise the 'FCounter' will be increased. If the 'FCounter' crosses the 'Cthreshold', the corresponding AP directly qualifies for TXOP, where as the APs in the 'Active' mode remain in 'Silent' mode until the beacon duration.

A fundamental diagram is given in Fig.4, where the 'Active' and 'Silent' mode of APs are presented.

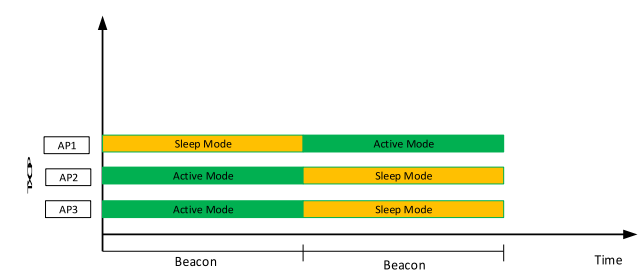

Fig. 4. 'Active' and 'Silent' mode of operations for AP1, $N_{1}<I_{2}+I_{3}$, $\mathrm{AP} 2, N_{2}>I_{1}+I_{3}$ and AP3, $N_{3}>I_{1}+I_{2}$

\section{Concurrent transmissions}

After winning the TXOP, the 'Active' mode APs need to decide which clients within the network are to be served concurrently. Obviously, the APs who have remaining degree of freedom greater than 2 after TXOP, will have to make this decision. Since the traffic is bursty in nature, with the clients joining and leaving the network, the best choice of the clients appropriate for the APs depend on parameters such as network capacity increment, fairness and or both depending on the scenario. Given the queues of uplink and downlink traffic, without loss of generality, we focus on the downlink.

Provided equal fairness among the clients, one can always serve the clients based on FIFO packet queues. The first packet will be served first and the remaining will be served accordingly. This method will ensure fairness, however, we can say nothing about the increment and the decrement of throughput capacity of the network. Another method could be a brute force approach, where all combinations of clients with queued packets are considered and the best combinations that would maximize the throughput are chosen. Though this method favors network capacity, it would undoubtedly ends up with deep unfairness among the clients who cannot maximize the throughput. We can also choose the best of the two methods at any moment, where the fairness and the throughput issue are checked. To prevent starvation of the clients, we always pick up the first packet in the queue and use a randomized design that exploits the best of the two 
choices, a standard approach for reducing the complexity of combinatorial problems [13].

\section{PERFORMANCE EVALUATION}

\section{A. Feasibility from $P H Y$}

We checked the feasibility of the solution of our PHY in a hardware platform made of Universal Software Radio Peripheral2 (USRP2) [14], RFX2400 daughter-boards and Jacksion labs equipments. The detail description of our PHY solution is presented in [15], thus, we only present the main result of it. ${ }^{3}$ The experimental result in Fig.5 showed that there is an average of about 5-6 dB gain in SNR per subcarriers due to our PHY solution. Besides, the collision free transmission is also shown which is the upper bound that our PHY solution is supposed to achieve. Despite imperfections in nulling caused by hardware offsets and other implementation limitations, the SNR gain of our PHY solution still possesses an acceptable performance of about $6 \mathrm{~dB}$ on an average. Clearly, the gain in SNR is about $10 \mathrm{~dB}$ in comparison to transmission in the HT scenario.

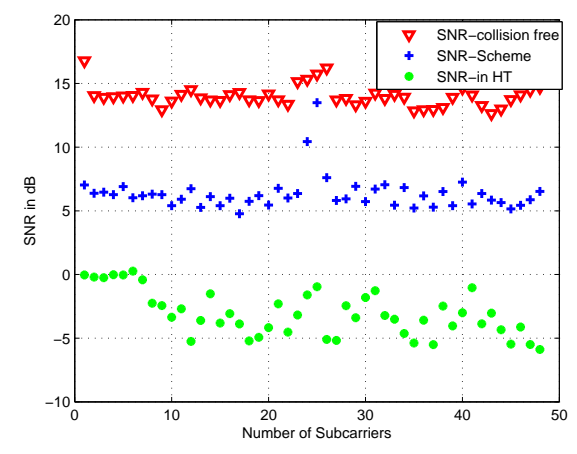

Fig. 5. SNR per subcarrier comparison with collision free Bob's transmission, our PHY scheme and in the HT condition

\section{B. Signaling overhead and Capacity gain of MAC}

We compare the signaling overhead of our protocol with the traditional RTS/CTS mechanism for handling the hidden terminal in WLANs. We further compare the total network capacity gain of the network of our protocol with the traditional RTS/CTS. As shown in Fig.3, the payload is not transmitted until we get all the channels and calculate the precoding vectors for APs. This period is defined as the signaling period.

In a typical HT scenario for 2 networks, we calculated the signaling overheads associated with different frames as shown in Fig.3. Since a PLCP preamble and a PLCP header are added to an MPDU to create a PLCP Protocol Data unit (PPDU), the transmission duration of the NDP announce frame is given by $T_{N D P_{a n n}}=$ PLCP frame $+\left[\frac{25}{B p S(m)}\right]$. tSymbol $=$ $40+\left[\frac{25}{3}\right] .4 \mu s=73.33 \mu s$. The PLCP frame is the compatible

\footnotetext{
${ }^{3}$ The standard GNURadio libraries [16], C++ and python were used in the Ubuntu 11.04 environment as software. The experiment was carried out in the indoor environment with operating frequency of $2.45 \mathrm{GHz}$, FFT length 64 and occupied subcarriers 48 .
}

frame with legacy standards and is define in IEEE802.11ac standard, whereas $t$ Symbol $=4 \mu \mathrm{s}$ is the OFDM symbol interval. The type of modulation used is BPSK with data rate $6 \mathrm{Mbps}$ and code rate $\frac{1}{2}$. The VHT NDP frame has the same format as the VHT PPDU except the data field, so the transmission duration $T_{N D P}=(8 \times 5)=40 \mu \mathrm{s}$. Likewise, the time duration of Beamforming Report Poll is $T_{B R_{\text {Poll }}}=40+\left[\frac{20}{3}\right] .4 \mu s=66.67 \mu \mathrm{s}$. We calculated the time duration of VHT Compressed beamformimg assuming payload length $l=200$ octets, $T_{C B_{\text {Report }}}=40+\left[\frac{5+l}{3}\right] .4 \mu s=313.33 \mu \mathrm{s}$. Thus, for typical two network, $K=2$, the signaling overhead is given by $T_{O H}=T_{N D P_{a n n}}+T_{N D P}+2 \times T_{C B_{\text {Report }}}=+$ $T_{B R_{\text {Poll }}}+5 \times S I F S=886.66 \mu \mathrm{s}$, where $t S I F S=16 \mu \mathrm{s}$. The traditional signaling overhead for RTS/CTS scheme is given by $T_{R T S / C T S}=T_{D I F S}+T_{R T S}+T_{C T S}+2 \times S I F S=$ $(34+50.33+42.33+2 \times 16)=158.7 \mu s$.

Based on the calculations of signaling overhead of our MAC protocol, we studied the impact of signaling overhead on the capacity of the network. The simulations are carried out for a typical scenario with 6 antenna AP2 from $i$ th network who wins the TXOP and has 2 clients from the $j$ th network in its transmission range. Thus, there are 4 clients inside the network to be served concurrently. ${ }^{4}$

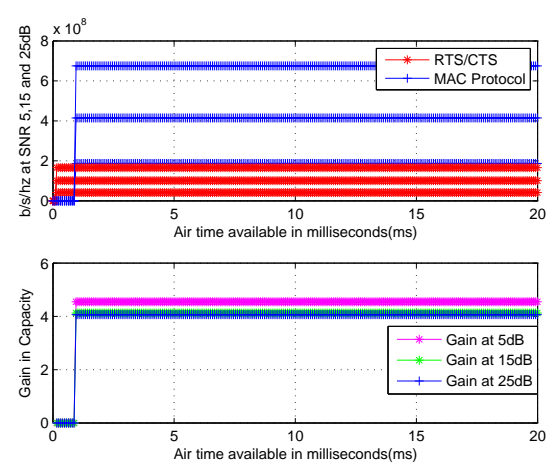

Fig. 6. Capacity comparision between RTS/CTS and our MAC protocol, air-time $20 \mathrm{~ms}$
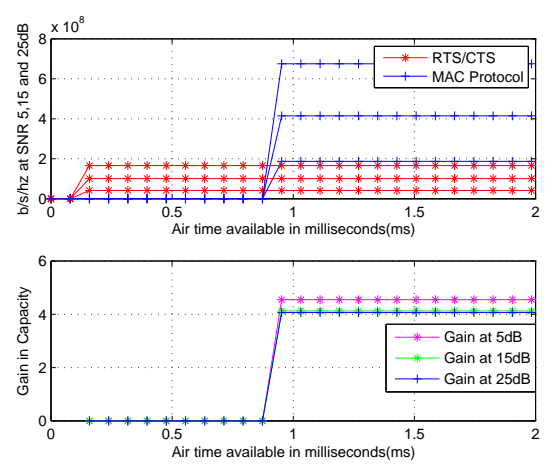

Fig. 7. Capacity comparision between RTS/CTS and our MAC protocol, air-time $2 \mathrm{~ms}$

\footnotetext{
${ }^{4}$ Typically, $N=6$ and $I=2$ (from the same network), so concurrent transmissions after TXOP is $N-I=4$.
} 
We first took an arbitrary air time $t=20 \mathrm{~ms}$ and compared the capacity gain for our MAC and RTS/CTS at 5, 15 and $25 \mathrm{~dB}$ respectively. Our simulation result in Fig.6 reveals that RTS/CTS scheme has an early gain in capacity at around $157.8 \mu \mathrm{s}$ whereas our MAC protocol does not gain in capacity until $886.65 \mu \mathrm{s}$. This was an expected behavior as our MAC protocol has higher signaling overhead than the RTS/CTS scheme. However, interestingly, we observed that our MAC protocol has about 4-5 times capacity gain compared to RTS/CTS scheme. Normally, lower signaling overhead is desired so that the available transmission time can be better utilized for packet transmissions. However, despite having higher signaling overhead than RTS/CTS, our MAC protocol has significant network capacity gain. This may look contradictory to the first statement, however, the gain in capacity comes from the concurrent transmissions (made possible by precoding vector) that takes place once the handshaking process is completed. Thus, the long signaling time in our MAC protocol is compensated by the capacity gain contributed by concurrent transmissions. In summary, despite larger signaling overhead compared to RTS/CTS, our MAC protocol have a significant capacity gain in the network.

Furthermore, we have a closer look with a short available air time $t=2 \mathrm{~ms}$ with all other factors remaining the same. Fig. 7 shows that we have a capacity gain of around 4-5 times which is the same gain as in $t=20 \mathrm{~ms}$. From Fig.6 and Fig.7 it is clear that the gain holds for all the available air time satisfying $t>886.65 \mu \mathrm{s}$. Thus, irrespective to the available air time $t>886.65 \mu \mathrm{s}$, the constant gain in the range of 4-5 times with respect to the traditional RTS/CTS is achieved by our MAC protocol at 5, 15 and $25 \mathrm{~dB}$ respectively.

\section{Performance of the concurrency algorithm}

We checked the throughput of the AP which has $(N-I=$ $6-2=4) 4$ concurrent transmissions to maintain, against three approaches discussed in Section $I V, D$. The simulation study shows that out of the three, Brute Force has higher throughput followed by the Best of the two choices and FIFO. Empirically, the fairness index among the client is in the reverse order to the network capacity of the algorithm.

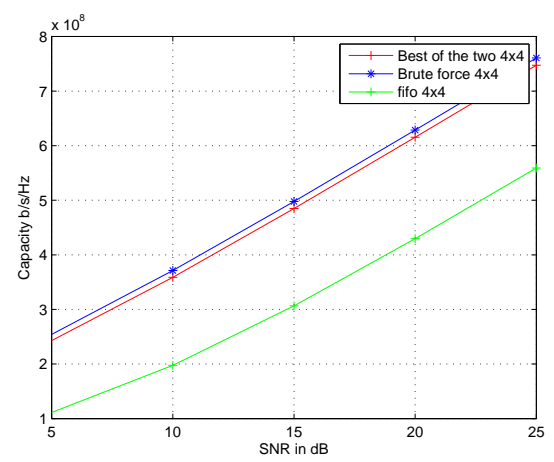

Fig. 8. Throughput comparison among FIFO, Brute Force and Best of the two approach

\section{CONCLUSION}

Collision of signals due to the HT are ineluctable in MUMIMO WLANs. This paper presents our MAC layer design to solve the Hidden Terminal problem while taking care of network capacity, fairness and signaling overhead. The Zeroforcing precoding solution at the PHY layer is seen effective to deal with the HT problem as we see the received SNR gain from 5-11dB in our USRP2/GNURadio prototype setting while our MAC layer design is able to maintain a constant network capacity gain of 4-5 times, compared to RTS/CTS, makes fair deal among APs for TXOP and compensates signaling overhead.

\section{REFERENCES}

[1] [Online]. Available: http://www.infonetics.com/pr/2013/2Q13-WirelessLAN-Market-Highlights.asp

[2] Y.-C. Cheng, J. Bellardo, P. Benkö, A. C. Snoeren, G. M. Voelker, and S. Savage, "Jigsaw: Solving the puzzle of enterprise 802.11 analysis," in Proceedings of the 2006 Conference on Applications, Technologies, Architectures, and Protocols for Computer Communications, ser. SIGCOMM '06. New York, NY, USA: ACM, 2006, pp. 39-50. [Online]. Available: http://doi.acm.org/10.1145/1159913.1159920

[3] M. Bertocco, G. Gamba, A. Sona, and S. Vitturi, "Performance measurements of csma/ca-based wireless sensor networks for industrial applications," in Instrumentation and Measurement Technology Conference Proceedings, 2007. IMTC 2007. IEEE, May 2007, pp. 1-6.

[4] X. Yang and N. Vaidya, "On physical carrier sensing in wireless ad hoc networks," in INFOCOM 2005. 24th Annual Joint Conference of the IEEE Computer and Communications Societies. Proceedings IEEE, vol. 4. IEEE, 2005, pp. 2525-2535.

[5] F. A. Tobagi and L. Kleinrock, "Packet switching in radio channels: part ii-the hidden terminal problem in carrier sense multiple-access and the busy-tone solution," Communications, IEEE Transactions on, vol. 23, no. 12 , pp. $1417-1433,1975$.

[6] C. Ware, J. Judge, J. Chicharo, and E. Dutkiewicz, "Unfairness and capture behaviour in 802.11 adhoc networks," in Communications, 2000. ICC 2000. 2000 IEEE International Conference on, vol. 1. IEEE, 2000, pp. $159-163$.

[7] A. Sheth, C. Doerr, D. Grunwald, R. Han, and D. Sicker, "Mojo: A distributed physical layer anomaly detection system for 802.11 wlans," in In Proceedings of MobiSys. ACM Press, 2006, pp. 191-204.

[8] P. Karn, "Maca-a new channel access method for packet radio," in ARRL/CRRL Amateur radio 9th computer networking conference, vol. 140, 1990, pp. 134-140.

[9] P. C. Ng, S. C. Liew, K. C. Sha, and W. T. To, "Experimental study of hidden node problem in ieee 802.11 wireless networks," Sigcomm Poster, p. 26, 2005.

[10] K. Lin, Y.-J. Chuang, and D. Katabi, "A light-weight wireless handshake," ACM SIGCOMM Computer Communication Review, vol. 42, no. 2, pp. 28-34, 2012.

[11] J. G. Andrews, "Interference cancellation for cellular systems: a contemporary overview," Wireless Communications, IEEE, vol. 12, no. 2, pp. 19-29, 2005.

[12] S. Gollakota and D. Katabi, Zigzag decoding: combating hidden terminals in wireless networks. ACM, 2008, vol. 38, no. 4.

[13] M. Mitzenmacher, "The power of two choices in randomized load balancing," Parallel and Distributed Systems, IEEE Transactions on, vol. 12, no. 10, pp. 1094-1104, 2001.

[14] [Online]. Available: http://www.ettus.com/

[15] S. Shrestha, G. Fang, E. Dutkiewicz, and X. Huang, "Addressing hidden terminals in wlans with zero forcing coordinated beamforming," in Proceedings of 14 th International Symposium on Communication and Information Technologies, 2014.

[16] [Online]. Available: http://www.gnu.org/ 